\title{
Anti-CD69 monoclonal antibody treatment inhibits airway inflammation in a mouse model of asthma*
}

\author{
Hui-ying $\mathrm{WANG}^{\dagger 1}$, Yu DAI ${ }^{2}$, Jiao-li WANG ${ }^{3}$, Xu-yan $\mathrm{YANG}^{4}$, Xin-guo JIANG ${ }^{5}$ \\ ( ${ }^{1}$ Department of Allergy, the Second Affiliated Hospital, School of Medicine, Zhejiang University, Hangzhou 310009, China) \\ ( ${ }^{2}$ Department of Clinical Laboratory, the Second Affiliated Hospital, School of Medicine, Zhejiang University, Hangzhou 310009, China) \\ ( ${ }^{3}$ Department of Respiratory Medicine, Hangzhou First People's Hospital, Hangzhou 310006, China) \\ $\left({ }^{4}\right.$ Department of Rheumatology and Clinical Immunology, the Second Affiliated Hospital, School of Medicine, \\ Zhejiang University, Hangzhou 310009, China) \\ ( 5 Department of Medicine, VA Palo Alto Health Care System/Stanford University School of Medicine, Stanford, CA 94305, USA) \\ "E-mail: marywang@zju.edu.cn \\ Received Oct. 23, 2014; Revision accepted Feb. 1, 2015; Crosschecked June 15, 2015
}

\begin{abstract}
Objective: Airway inflammation and airway hyper-responsiveness (AHR) are principle pathological manifestations of asthma. Cluster of differentiation 69 (CD69) is a well-known co-stimulatory factor associated with the activation, proliferation as well as apoptosis of immune cells. This study aims to examine the effect of anti-CD69 monoclonal antibody $(\mathrm{mAb})$ on the pathophysiology of a mouse model of asthma. Methods: A murine model of ovalbumin (OVA)-induced allergic airway inflammation was used in this study. Briefly, mice were injected with $20 \mu \mathrm{g}$ chicken OVA intraperitoneally on Days 0 and 14 , followed by aerosol provocation with $1 \%(0.01 \mathrm{~g} / \mathrm{ml})$ OVA on Days 24 , 25 , and 26. Anti-CD69 mAb or isotype IgG was injected intraperitoneally after OVA challenge; dexamethasone (DXM) was administrated either before or after OVA challenge. AHR, mucus production, and eosinophil infiltration in the peribronchial area were examined. The levels of granulocyte-macrophage colony-stimulating factor (GM-CSF) and interleukin-5 (IL-5) in bronchoalveolar lavage fluid (BALF) were also assayed as indices of airway inflammation on Day 28 following OVA injection. Results: Pretreatment with DXM together with anti-CD69 mAb treatment after OVA provocation completely inhibited AHR, eosinophil infiltration and mucus overproduction, and significantly reduced BALF IL-5. However, treatment with DXM alone after OVA challenge only partially inhibited AHR, eosinophil infiltration and mucus overproduction, and did not diminish BALF IL-5. Treatment with either DXM or anti-CD69 mAb did not alter the concentration of BALF GM-CSF. Conclusions: Anti-CD69 mAb treatment inhibits established airway inflammation as effectively as DXM pretreatment. This study provides a potential alternative therapeutic opportunity for the clinical management of asthma and its exacerbation.
\end{abstract}

Key words: Cluster of differentiation 69 (CD69), Eosinophil, Interleukin-5 (IL-5), Asthma doi: $10.1631 /$ jzus.B1400285

Document code: A

CLC number: R562.2

\section{Introduction}

Asthma is a chronic airway inflammation, characterized by the infiltration of inflammatory cells (in-

\footnotetext{
* Project supported by the National Natural Science Foundation of China (No. 30600266), the Zhejiang Provincial Science and Technology Project (No. 2011C37073), the Zhejiang Provincial Natural Science Foundation (No. LQ12H16012), the National Key Clinical Project of Allergy of China, and the National Key Clinical Specialist Construction Programs of China

(D) ORCID: Hui-ying WANG, http://orcid.org/0000-0001-8630-2592

(C) Zhejiang University and Springer-Verlag Berlin Heidelberg 2015
}

cluding mast cells and eosinophils etc.), mucus production, and airway hyper-responsiveness (AHR) (Busse and Lemanske, 2001). T helper 2 (Th2) lymphocytes and related cytokines are thought to have crucial roles in driving the pathophysiological changes of asthmatic airways (McGee and Agrawal, 2006). The therapeutic goal for asthma is to attenuate/eliminate the inflammatory reaction by reducing/eliminating tissue inflammatory cell infiltration and to diminish AHR.

Cluster of differentiation 69 (CD69), a type II membrane antigen, was discovered about 20 years 
ago. It is also known as an early activation marker for several kinds of leukocyte. Resting peripheral leukocytes do not express CD69. However, leukocyte infiltrates have been reported to express CD69 in various pathological conditions such as chronic inflammation (Wang et al., 2006; Mohammadi Nejad et al., 2013), infectious diseases (Iwashiro et al., 2001), autoimmune disorders, and tumors (Atzeni et al., 2004; Epardaud et al., 2008).

The detailed role of CD69 in the immune response is not completely understood, mainly because no known ligand has been identified. Traditionally, CD69 is considered a co-stimulatory factor for cell activation and/or proliferation (Lewkowich et al., 2005). Engagement of CD69 has been shown to induce an increase of intracellular $\mathrm{Ca}^{2+}$, synthesis of cytokines and their receptors, expression of the protooncogene c-myc and c-fos, as well as enhanced cellular proliferation (Cebrian et al., 1988; Santis et al., 1992; Tugores et al., 1992; Testi et al., 1994; Unutmaz et al., 1994). Through CD69 targeting and deficiency experiments, Alari-Pahissa et al. (2012) revealed that CD69 might have multiple immunoregulatory roles. $\mathrm{CD} 69$ has also been thought to play important roles in the generation and maintenance of professional memory Th lymphocytes (Shinoda et al., 2012). $\mathrm{CD}^{+} 9^{+} \mathrm{T}$ cells were demonstrated to induce indoleamine 2,3-dioxygenase in tumor-associated macrophages and hence to down-regulate inflammatory immune responses (Zhao et al., 2012). Other studies showed that CD69 expression is associated with increased incidence and enhanced severity of autoimmune and inflammatory diseases including collagen II-induced arthritis (Sancho et al., 2003), allergic airway inflammation and skin contact hypersensitivity (Martin et al., 2010b), and autoimmune myocarditis (Cruz-Adalia et al., 2010). In addition, $\mathrm{CD} 9^{-/-}$mice appeared to be more susceptible to Listeria monocytogenes infection, an effect probably associated with the enhancement of type I and II interferon responses (Vega-Ramos et al., 2010).

Based on these previous studies, CD69 is considered as a potential therapeutic target for a number of chronic inflammatory diseases including allergic asthma. However, studies focusing on elucidating the effects of anti-CD69 monoclonal antibody (mAb) treatment in allergic airway inflammation are conflicting (Miki-Hosokawa et al., 2009; Martin et al., 2010b). In the present study we investigated the effects of anti-CD69 mAb treatment on established airway eosinophilia and mucus production as well as AHR in asthmatic mice. We also compared its effects to dexamethasone (DXM) administrated before or after OVA challenge. Our study suggested that anti-CD69 mAb may be therapeutically effective in inhibiting airway inflammation and AHR of bronchial asthma.

\section{Materials and methods}

\subsection{Animals}

We purchased C57BL/6 mice from the Experimental Animal Center of Zhejiang University, Hangzhou, China; the mice were raised in microisolator cages in specific pathogen-free animal facilities. Male mice at 8-12 weeks of age were used for experiments. Experimental protocols were approved by the Ethical Committee for Animal Studies of Zhejiang University, Hangzhou, China.

\subsection{Ovalbumin (OVA) sensitization/aerosol chal- lenge and administration of anti-CD69 $\mathrm{mAb}$ and DXM}

Mouse models of asthma were prepared with chicken OVA sensitization and challenge. Briefly, $20 \mu \mathrm{g}$ $(100 \mu 1)$ chicken OVA that was emulsified with alum (2.25 mg Al( $(\mathrm{OH})_{3}, 2 \mathrm{mg} \mathrm{Mg}(\mathrm{OH})_{2}$; Pierce, USA) was injected into the mice intraperitoneally on Days 0 and 14. The airway challenge was provided by an aerosol of saline alone or $1 \%(0.01 \mathrm{~g} / \mathrm{ml})$ OVA in saline generated by ultrasonic nebulization (DeVilbiss, USA), which the mice inhaled for 20 min on Days 24, 25, and 26. DXM $(5 \mathrm{mg} / \mathrm{ml}$; Jinyao Group, Co., Ltd., Tianjin, China) was dissolved in saline and injected intraperitoneally at a dose of $2 \mathrm{mg} / \mathrm{kg}$ on Days 21 and 23 (before aerosol challenge) or on Day 26 (6 h after aerosol challenge) and Day 27. A total of $50 \mu \mathrm{g}$ hamster anti-mouse CD69 mAb (H1.2F3, R\&D Systems, USA) or hamster anti-mouse isotype IgG (R\&D Systems, USA) in $100 \mu$ l phosphate-buffered solution (PBS) was injected intraperitoneally on Day 26 (6 h after aerosol provocation) and Day 27. Airway eosinophils infiltration, mucus production, cytokine levels of bronchoalveolar lavage fluid (BALF), and AHR were measured on Day 28 to assess the physiopathologic changes in asthmatic mice, as summarized in Fig. 1. 


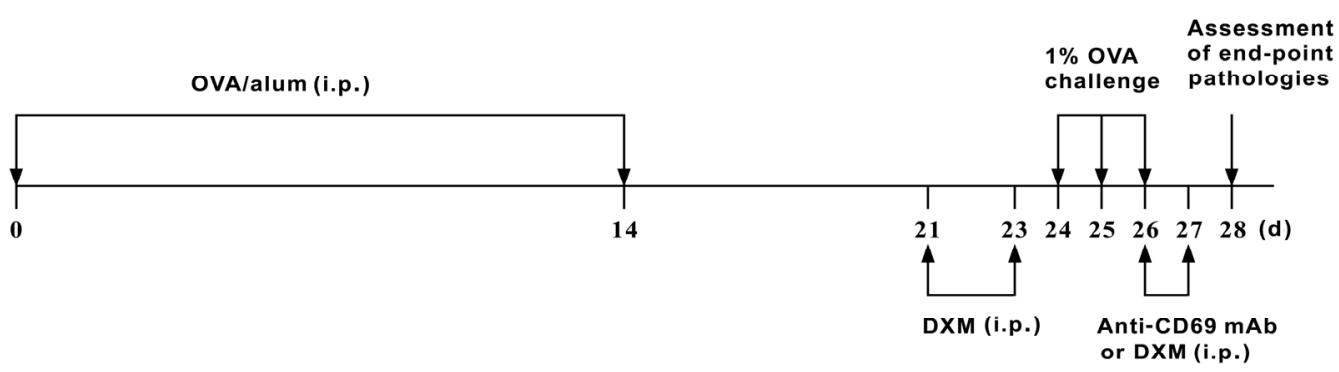

Fig. 1 Time line presentation of the OVA protocol and the intraperitoneal (i.p.) administration of DXM and anti-CD69 mAb

\subsection{Measurement of AHR under challenge of methacholine}

Airway flow obstruction of mice in response to methacholine aerosol was measured via whole-body plethysmography as the index of AHR (Lee et al., 1997). In this non-invasive methodology (Buxco Electronics, Troy, USA), unrestrained and conscious mice were in one chamber; airway physiologic data, such as enhanced pause (Penh), minute volume, breathing frequency, and tidal volume, were extrapolated from the pressure differences between this chamber and its neighboring reference chamber. Penh is the sum of total pulmonary airflow including the upper and lower respiratory tract airflows in each respiratory cycle. It was established to be close to that measured using traditional invasive techniques on ventilated animals (Hamelmann et al., 1997) for airway resistance assessment. Thus Penh was used here as a dimensionless parameter for lung function in mice. The ratio of dose-response Penh of methacholine $(\mathrm{mg} / \mathrm{ml})$ to baseline Penh was compared among different groups of mice, and then plotted against $\log _{10}$ of methacholine solution.

2.4 Measurements of airway inflammation: eosinophil infiltration and OVA-induced histopathologic changes in the lung

The cellular infiltration of the bronchial lumens was calculated as previously reported (Borchers et al., 2001). Lungs of mice were lavaged intratracheally with $0.5 \mathrm{ml}$ of PBS (Gibco BRL, USA) containing $2 \%$ fetal calf serum (FCS) twice. Recovered fluid from each mouse was then collected and centrifuged. The supernatant was stored at $4{ }^{\circ} \mathrm{C}$ and the cell deposits were washed and counted with a hemacytometer. Cytospin slides were subsequently made using a cytospin machine (Shandon Scientific, USA). Four hundred cells were counted and eosinophils were determined by Diff-Quik stain (Baxter Healthcare, USA). Hematoxylin/eosin (H\&E) staining of the lung tissue $(4 \mu \mathrm{m})$ was used to determine eosinophil infiltration. The number of tissue-infiltrated eosinophils was quantified using software for image analysis (Image Pro Plus, Silver Spring, USA). The density of eosinophils in the lung tissue was calculated from the total number of eosinophils infiltrated into the peribronchial area divided by the total number of bronchioles.

The amount of mucus in the epithelial cells, as the index of the histopathology of the airways, was assessed through paraffin-embedded sections. Mucus production as well as goblet cell metaplasia was measured on parasagittal sections $(4 \mu \mathrm{m})$ with periodic acid-Schiff's (PAS) stain and hematoxylin/ methyl green counter-stain. The same image analysis software was used to assess both proximal and distal airway mucus productions via bright field microscopy (five mice in each group). The mucus index was defined as the sum of mucus produced per bronchiole divided by the number of bronchioles (Justice et al., 2002). An experienced pathologist assessed all samples in a blind manner.

\subsection{Cytokine assays}

Mouse interleukin-5 (IL-5) and granulocyte macrophage colony-stimulating factor (GM-CSF) levels in BALF were assayed using enzyme-linked immunosorbent assay (ELISA) kits (R\&D Systems, USA) under the manufacturer's instructions. The limits of detection for GM-CSF and IL-5 were $7 \mathrm{pg} / \mathrm{ml}$.

\subsection{Statistical analysis}

Data were expressed as mean \pm standard error (SE). Differences between multi-groups were tested 
with one-way analysis of variance (ANOVA) or the Mann-Whitney test if the data were not in a Gaussian distribution. Student's $t$-test or Wilcoxon rank-sum test was performed to compare the difference between two groups. $P<0.05$ was considered significant. SigmaStat 3.5 software (Systat Software Inc., San Jose, CA, USA) was used for all statistical analyses.

\section{Results}

\subsection{Anti-CD69 mAb eliminates airway eosinophil infiltration}

A hallmark of airway inflammation in the asthmatic model is eosinophil infiltration (Busse and Lemanske, 2001). We first examined the effect of anti-CD69 $\mathrm{mAb}$ on airway infiltration by eosinophil cells. Analysis of the BALF showed that anti-CD69 $\mathrm{mAb}$ administration completely abolished eosinophil cell migration into the airway lumen, an effect that is comparable to DXM therapy (Fig. 2). We next examined eosinophil cell infiltration surrounding the airways (Fig. 3). H\&E staining showed that OVA administration induced massive infiltration of eosinophils around the airways (Fig. 3a). As expected, DXM treatment effectively prevented or inhibited peribronchial eosinophil infiltration (Figs. 3c and 3d). Comparable to DXM treatment, anti-CD69 mAb, but not isotype $\operatorname{IgG}$, completely inhibited peribronchial eosinophil infiltration (Figs. 3e and 3f). Eosinophil infiltration was further quantified (Fig. 3g). Together, these data suggested that anti-CD69 mAb suppresses eosinophil accumulation in the peribronchial area as well as migration into the airway lumen.

\subsection{Anti-CD69 mAb inhibits OVA-induced airway epithelial mucus production}

Pathological airway changes following OVA sensitization/provocation include hyperplasia of epithelial cells, mucus overproduction, discontinuous epithelium, thickened basal layer, and hypertrophy of smooth muscle. Consistent with our earlier findings (Wang et al., 2006), OVA administration induced airway mucus accumulation (Figs. 4a and 4b). DXM, as a known effective therapy, alleviated or inhibited mucus production (Figs. 4c and 4d). Anti-CD69 mAb nearly totally abolished mucus accumulation (Figs. 4e and 4f). Quantification of mucus production showed that anti-CD69 mAb treatment is as effective as DXM pretreatment in reducing mucus production (Fig. 4g). These data further suggest that anti-CD69 mAb is effective in reversing pathological changes associated with allergic airway inflammation.

\subsection{Anti-CD69 mAb diminishes BALF IL-5 levels}

Cytokines in BALF are known to be associated with allergic airway inflammation, especially Th2 cytokines. IL-5 and GM-CSF are important growth factors that enhance the survival and activation of eosinophils in airway lumen (Su et al., 2008; Takatsu and Nakajima, 2008; Sheikh Bahaie et al., 2010). In this model of allergic airway inflammation, no significant GM-CSF changes were observed following OVA challenge as well as with DXM or anti-CD69 $\mathrm{mAb}$ treatment (Fig. 5a). On the other hand, IL-5 levels were significantly elevated following OVA induction of airway inflammation, and anti-CD69 $\mathrm{mAb}$ was able to reduce BALF IL-5 levels dramatically,

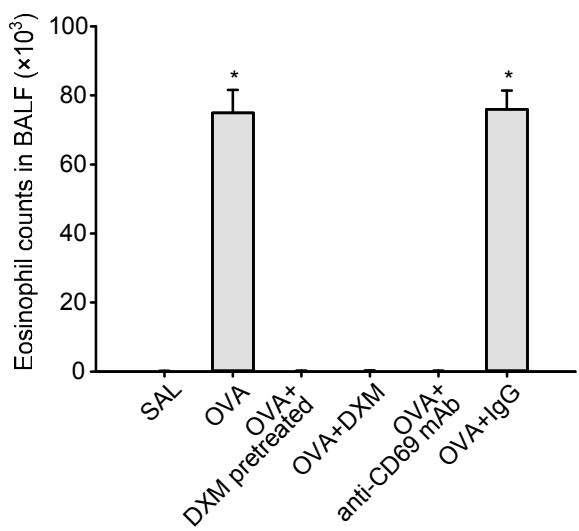

Fig. 2 Effect of anti-CD69 mAb or DXM on eosinophil infiltration in BALF

Mice were divided into six groups: saline-sensitized/ challenged mice (SAL); OVA-sensitized/challenged mice (OVA); OVA-sensitized/challenged mice with DXM pretreatment before OVA challenge (OVA+DXM pretreated); OVA-sensitized/challenged mice treated by DXM after OVA challenge (OVA+DXM); OVA-sensitized/challenged mice treated by anti-CD69 mAb after OVA challenge (OVA+ anti-CD69 $\mathrm{mAb}$ ); and OVA-sensitized/challenged mice with isotype $\operatorname{IgG}$ administration (OVA+IgG) (the same for the following Figs. 3-6). OVA sensitization/challenge induced obvious eosinophil infiltration in bronchial lumen, which was inhibited completely by anti-CD69 mAb or DXM treated either before or after OVA challenge. Eosinophils in the BALF of each animal cohort are expressed as the product of the total number of cells recovered. The values are presented as mean $\pm \mathrm{SE}$ derived from two sections per mouse ( $n=5-10$ mice per group). ${ }^{*} P<0.05$, compared with the SAL group, using Student's $t$-test 

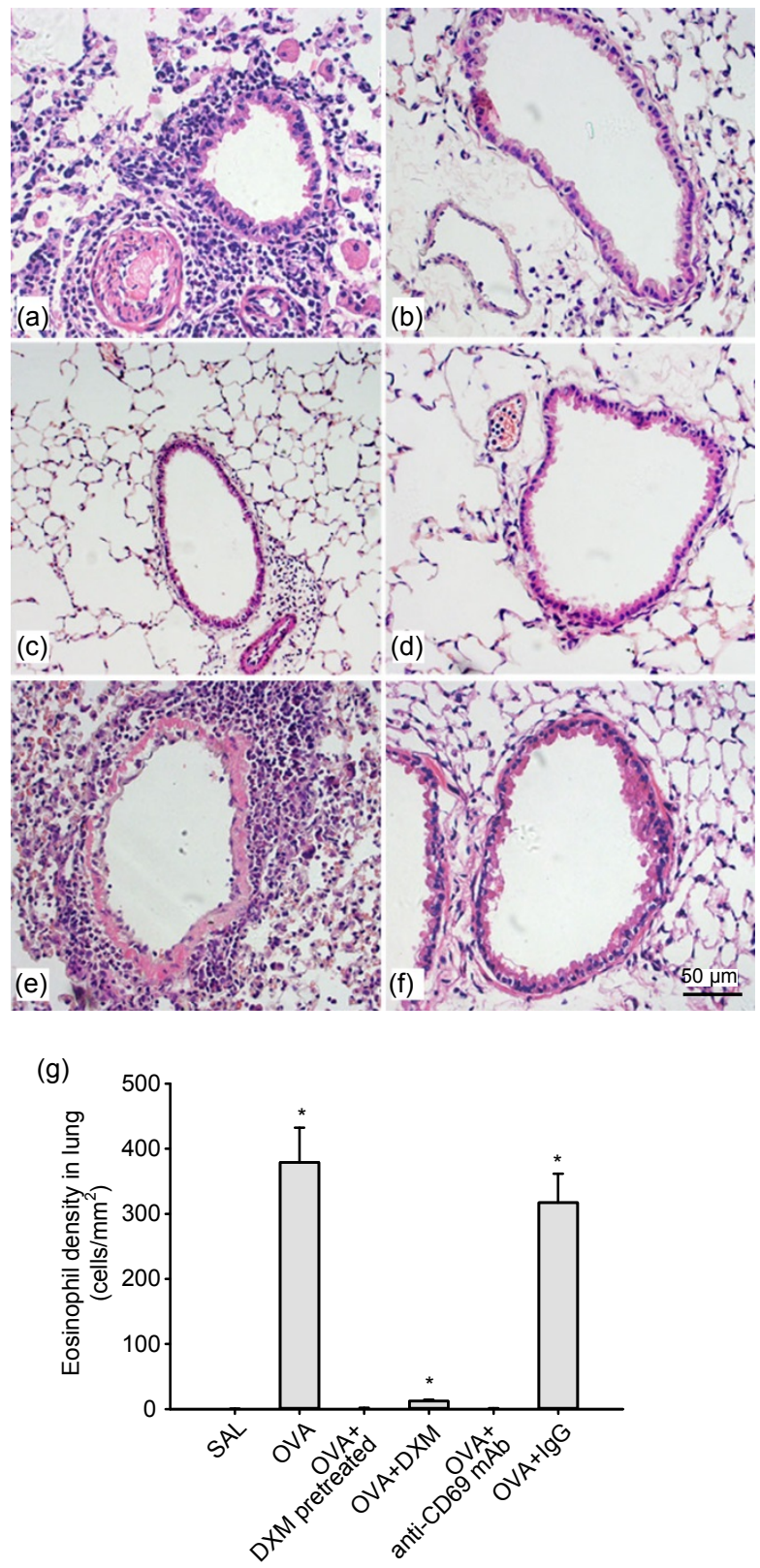

Fig. 3 Effect of anti-CD69 mAb or DXM on airway eosinophil distribution in asthmatic mice

The accumulation of eosinophils in the lungs of OVAtreated mice was assessed by H\&E staining. OVA administration induced massive eosinophil infiltration in peribronchial area, which was inhibited completely by anti-CD69 $\mathrm{mAb}$ or DXM treatment before OVA challenge, but partially by DXM treatment after OVA challenge. (a) OVAsensitized/challenged mice; (b) Saline-treated mice; (c) OVAsensitized/challenged mice with DXM treatment after OVA challenge; (d) OVA-sensitized/challenged mice with DXM treatment before OVA challenge; (e) OVA-sensitized/ challenged mice with isotype IgG administration; (f) OVAsensitized/challenged mice with anti-CD69 mAb administration. (g) Density of eosinophils in the lung tissue. ${ }^{*} P<0.05$, compared with the SAL group, using Student's $t$-test

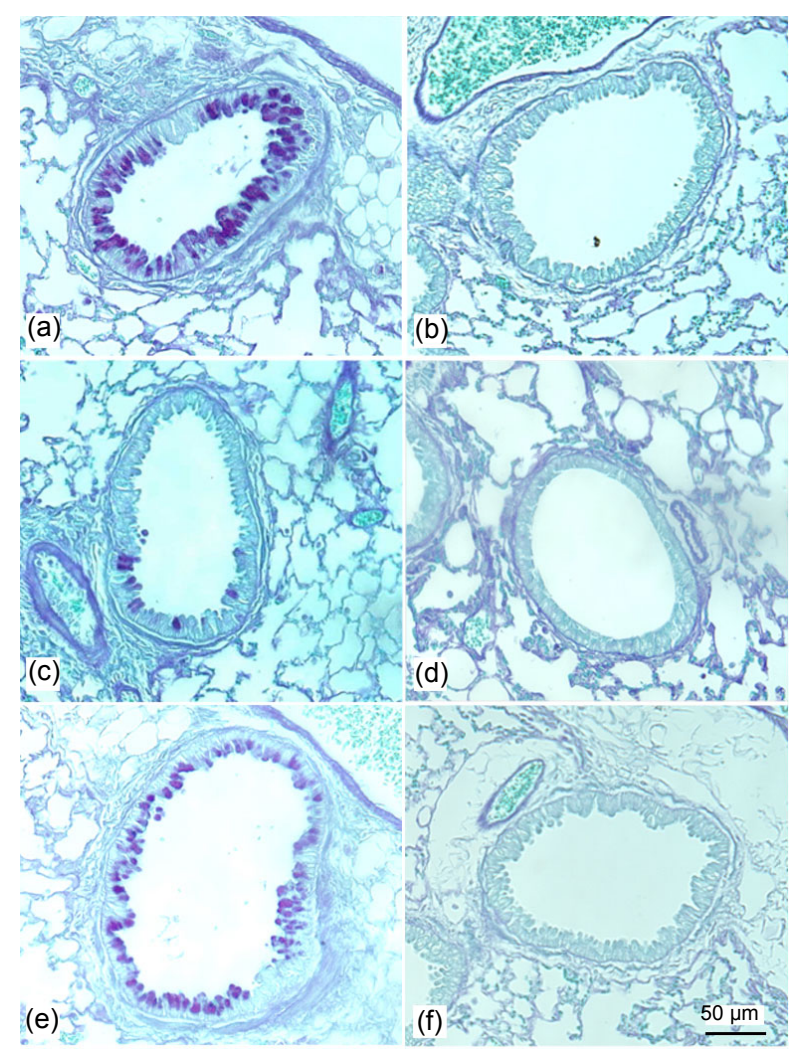

(g)

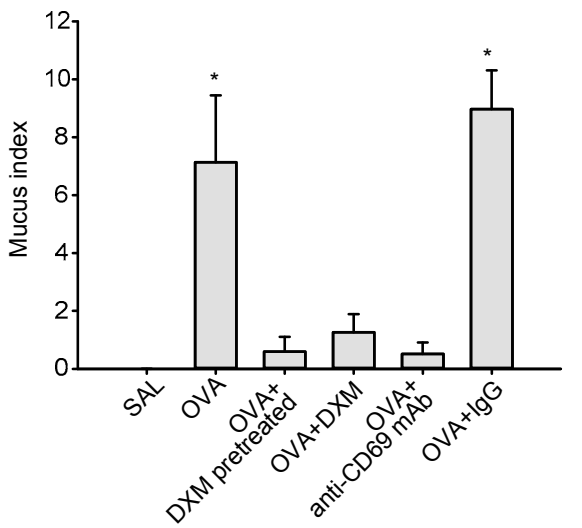

Fig. 4 Effect of anti-CD69 mAb or DXM on OVAinduced mucus overproduction in asthmatic mice

The administration of anti-CD69 mAb or DXM inhibits OVA-induced mucus over-production. Lung sections from the formalin-fixed/paraffin-embedded tissue were stained for the presence of mucin with periodic acid-Schiff's reagent (counter-stain: hematoxylin). The photographs presented show representative examples of the staining that occurs in bronchioles. (a) OVA-sensitized/challenged mice; (b) Salinetreated mice; (c) OVA-sensitized/challenged mice with DXM treatment after OVA challenge; (d) OVA-sensitized/ challenged mice with DXM treatment before OVA challenge; (e) OVA-sensitized/challenged mice with isotype IgG administration; (f) OVA-sensitized/challenged mice with anti-CD69 mAb administration. (g) Quantified assessment of mucus production of mice airways. ${ }^{*} P<0.05$, compared with the SAL group, using Student's $t$-test 
(a)

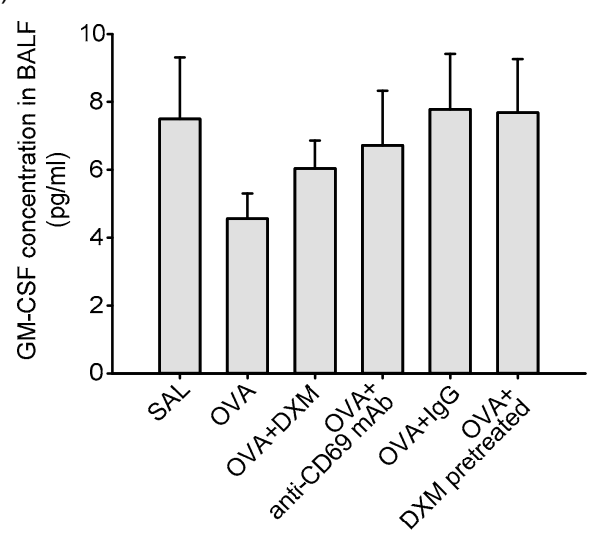

(b)

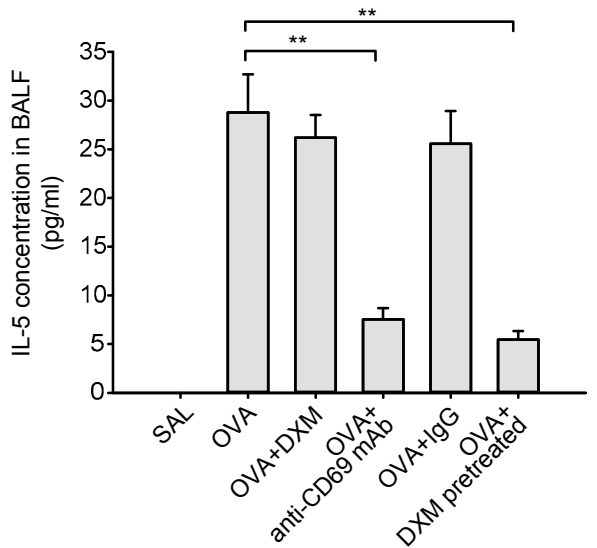

Fig. 5 Effects of anti-CD69 mAb or DXM pretreatment on GM-CSF (a) and IL-5 (b) in BALF of OVA-treated mice Anti-CD69 mAb or DXM pretreatment results in a decrease of IL-5 in BALF of OVA-treated mice. DXM administrated after OVA challenge makes no effect on IL-5. Expression of GM-CSF in BALF was not changed by OVA or anti-CD69 mAb or $\mathrm{DXM}$. The values are presented as mean $\pm \mathrm{SE}$ ( $n=5-10$ mice per group). ${ }^{* *} P<0.001$, compared with the SAL group, using Student's $t$-test

an effect that is similar to DXM pretreatment (Fig. 5b). Interestingly, late therapy with DXM was not able to reverse IL-5 levels (Fig. 5b), in contrast to its effect on reducing airway eosinophil cell infiltration and mucus production (Figs. 3 and 4). These data suggest that anti-CD69 antibody may have its unique advantages in inhibiting allergic airway inflammation.

\subsection{Anti-CD69 mAb completely inhibits allergen- induced AHR}

We next evaluated the effect of anti-CD69 mAb on AHR, another hallmark of asthma. Penh values were assessed with whole-body plethysmography $48 \mathrm{~h}$ after the last OVA challenge. Our results showed that anti-CD69 $\mathrm{mAb}$ was as effective as DXM pretreatment in reducing methacholine-induced AHR, and interestingly, the anti-CD69 treatment is even more effective than late DXM administration (Fig. 6).

\section{Discussion}

The present study demonstrated that therapeutic administration of anti-CD69 mAb efficiently eliminated established airway inflammation and AHR in asthmatic mice. In addition, our data also suggest that in several aspects, anti-CD69 antibody is more potent than late DXM treatment, the classic first line medicine for asthma treatment.

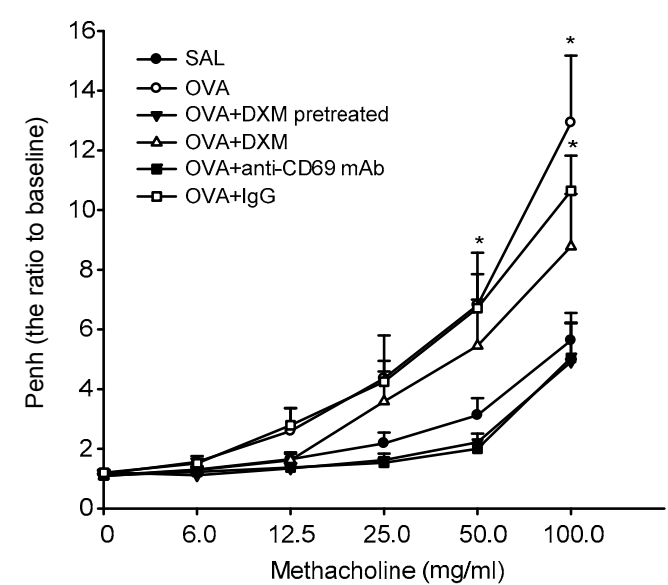

Fig. 6 Effect of anti-CD69 mAb on OVA-induced AHR OVA-induced AHR is inhibited by anti-CD69 mAb administrated after OVA challenge. Airway reactivity (percent baseline Penh) in response to increasing doses of nebulized methacholine was assessed by whole-body plethysmography ( $n=10$ mice per group). ${ }^{*} P<0.05$, compared with the SAL group, using Student's $t$-test

Because of its extensive effect on different types of immune cells, CD69 has been considered as a therapeutic target for many different diseases. However, the trials of anti-CD69 mAb in mouse asthmatic models by two other groups have led to completely different results. Martin et al. (2010b) found that anti-CD69 $\mathrm{mAb}$ and CD69 deficiency $\left(\mathrm{CD} 69^{-/-}\right)$ enhanced asthmatic inflammation, while MikiHosokawa et al. (2009) discovered that anti-CD69 $\mathrm{mAb}$ attenuated airway inflammation. 
The apparent contrasting results obtained by our group and others might be caused by differences in the antibody used or in the timing of antibody administration. Martin et al. (2010b) used mAb made in mouse, which is an anti-mouse CD69 mAb, while we and Miki-Hosokawa et al. (2009) used anti-CD69 $\mathrm{mAb}$ produced in hamster. These were completely different antibodies; the hamster-produced anti-CD69 has a much lower half-life in serum than the antiCD69 mAb used by Martin et al. (2010b). Another possible explanation is the pleiotropic function of CD69, which might have different effects on cell activation and apoptosis. CD69 has been shown to regulate Th17 cell differentiation through its cytoplasmic tail associated with the Jak3/Stat5 signalling pathway (Martin et al., 2010a), and inhibit the egress of lymphocytes from lymph nodes by degrading sphingosine 1-phosphate receptor-1 (S1P1), which is required for lymphocyte egress (Bankovich et al., 2010). In addition, it also induces apoptosis of activated lymphocytes (Pajusto et al., 2004). Thus, treatment with anti-CD69 mAb after allergen challenge resulted in the inhibition of inflammation, while the early blockade of CD69 expression (for example, by administration of anti-CD69 mAb at the start point of the sensitization process) might result in the opposite effect.

Anti-CD69 mAb might eliminate airway inflammation and AHR through two possible mechanisms. The first is the direct induction of apoptosis of $\mathrm{CD}^{+} \mathrm{T}$ cells and eosinophils in airways. Previous studies revealed that those activated $\mathrm{T}$ cells or eosinophils expressing high levels of CD69 are susceptible to spontaneous apoptosis in vitro (Matsumoto et al., 1998; Luttmann et al., 1999; Dallaire et al., 2003; Esplugues et al., 2003; Lewkowich et al., 2005; Wang et al., 2006). This pro-apoptotic effect of CD69 might be explained by the observation that CD69 is associated with the N-terminal fragment of calreticulin, a known apoptosis-inducing signal molecule (Vance et al., 2005). It is generally accepted that anti-CD69 mAb might enhance or induce the apoptosis of activated eosinophils despite the existence of IL-5 and GM-CSF (Walsh et al., 1996). Anti-CD69 $\mathrm{mAb}$-induced apoptosis is associated with caspase- 3 and caspase-8 activation (Al-Rabia et al., 2004) and TGF- $\beta$ production in the culture supplemented with IL-3, IL-5, or GM-CSF (Letterio and Roberts, 1998;
Al-Rabia et al., 2004). The second potential mechanism is that anti-CD69 mAb decreases the level of the Th2 cytokine, IL-5. IL-5 is thought to be a crucial regulator of eosinophil proliferation in asthma and other allergic disease (Shen et al., 2003). IL-5 has been shown to activate eosinophils to produce more IL-5 (Spencer et al., 2009), thus forming a positive feedback loop to expand the eosinophil reservoir in peripheral blood and bone marrow reacting to the allergen challenge. IL-5 also regulates the expression of genes involved in growth, survival, and effector function of eosinophils (Takatsu and Nakajima, 2008; Han and Mosher, 2014). Therefore, decreasing IL-5 levels in the local microenvironment may dramatically shorten the life span of $\mathrm{T}$ cells and eosinophils.

GM-CSF shares a common $\beta$ receptor subunit with IL-5, playing a key role in Th2 immunity regulation and eosinophil accumulation in the airway (Asquith et al., 2008; Su et al., 2008). Our data show that the concentration of GM-CSF is not affected by OVA sensitization and challenge, which is consistent with a study by van Rijt et al. (2002), suggesting that GM-CSF might be more important at the initial stages of allergic inflammation. However, the presence of GM-CSF did not affect the inhibition of inflammation caused by anti-CD69 mAb.

Extensive studies focusing on the elimination of eosinophils using different chemicals have been carried out, but few of these chemicals have led to promising clinical outcomes. Agents such as anti-IL-4 $\mathrm{mAb}$, anti-IL-5 mAb, and anti-CCR3 mAb blocked the formation of airway inflammation in animal models of asthma, but failed in clinical trials (Antoniu, 2009; Busse et al., 2010; Corren et al., 2010; Castro et al., 2011; Pavord et al., 2012). It is likely that in preclinical studies, these agents were effective as preventative therapy, but not necessarily able to reverse established disease. Anti-CD69 can effectively reverse established airway inflammation in mice; thus it may represent a promising therapy for human asthma.

Our study also showed that the therapeutic efficacy of anti-CD69 $\mathrm{mAb}$ for asthma is comparable to DXM. Glucocorticoids are powerful anti-inflammatory drugs, which cure disease by inhibiting pro-inflammatory cell recruitment and down-regulating the production of pro-inflammatory cytokines (Gauvreau et al., 2000). In most studies, DXM proves to be potent in blocking 
airway inflammation by intraperitoneal injection before antigen challenge or OVA sensitization/ challenge. Here, we have demonstrated the potent role of anti-CD69 $\mathrm{mAb}$ in eliminating eosinophils in airway lumen and the peribronchial area, mucus production and AHR after the inflammation was established. However, DXM treatment after OVA challenge is not as effective as pretreatment in eliminating peribronchial eosinophils and mucus production. One possibility is that it takes more time for DXM to reverse established inflammation, since DXM is not a short-acting agent. Another possibility is that the dosage of DXM is not enough to reverse established inflammation since a low dose has been found to inhibit only eosinophil inflammation while have no effect on AHR in the murine airway even before OVA challenge (Birrell et al., 2003). More dosage experiments are needed to prove this hypothesis. Therefore, DXM cannot function effectively in a microenvironment rich in all kinds of cytokine such as IL-5, IL-12, and IL-13, which increase the viability of eosinophils and limit the anti-inflammatory effect of DXM.

\section{Conclusions}

Treatment with anti-CD69 mAb could inhibit established airway inflammation of asthmatic mice as effectively as DXM. This study may provide a potential alternative regimen for the therapy of asthma and its exacerbation.

\section{Compliance with ethics guidelines}

Hui-ying WANG, Yu DAI, Jiao-li WANG, Xu-yan YANG, and Xin-guo JIANG declare that they have no conflict of interest.

All experimental protocols were approved by the Ethical Committee for Animal Studies of Zhejiang University, China. All institutional and national guidelines for the care and use of laboratory animals were followed.

\section{References}

Al-Rabia, M.W., Blaylock, M.G., Sexton, D.W., et al., 2004. Membrane receptor-mediated apoptosis and caspase activation in the differentiated EoL-1 eosinophilic cell line. J. Leukoc. Biol., 75(6):1045-1055. [doi:10.1189/ jlb.0803404]

Alari-Pahissa, E., Notario, L., Lorente, E., et al., 2012. CD69 does not affect the extent of $\mathrm{T}$ cell priming. PLOS ONE, 7(10):e48593. [doi:10.1371/journal.pone.0048593]

Antoniu, S.A., 2009. Mepolizumab for difficult-to-control asthma with persistent sputum eosinophilia. Expert Opin. Investig. Drugs, 18(6):869-871. [doi:10.1517/135437809 02922678]

Asquith, K.L., Ramshaw, H.S., Hansbro, P.M., et al., 2008. The IL-3/IL-5/GM-CSF common receptor plays a pivotal role in the regulation of Th2 immunity and allergic airway inflammation. J. Immunol., 180(2):1199-1206. [doi:10. 4049/jimmunol.180.2.1199]

Atzeni, F., del Papa, N., Sarzi-Puttini, P., et al., 2004. CD69 expression on neutrophils from patients with rheumatoid arthritis. Clin. Exp. Rheumatol., 22(3):331-334.

Bankovich, A.J., Shiow, L.R., Cyster, J.G., 2010. CD69 suppresses sphingosine 1-phosophate receptor-1 (S1P1) function through interaction with membrane helix 4. $J$. Biol. Chem., 285(29):22328-22337. [doi:10.1074/jbc. M110.123299]

Birrell, M.A., Battram, C.H., Woodman, P., et al., 2003. Dissociation by steroids of eosinophilic inflammation from airway hyperresponsiveness in murine airways. Respir. Res., 4(1):3. [doi:10.1186/rr197]

Borchers, M.T., Crosby, J., Farmer, S., et al., 2001. Blockade of CD49d inhibits allergic airway pathologies independent of effects on leukocyte recruitment. Am. J. Physiol. Lung Cell Mol. Physiol., 280(4):L813-L821.

Busse, W.W., Lemanske, R.F.J., 2001. Asthma. N. Engl. J. Med., 344(5):350-362. [doi:10.1056/NEJM20010201344 0507]

Busse, W.W., Katial, R., Gossage, D., et al., 2010. Safety profile, pharmacokinetics, and biologic activity of MEDI563 , an anti-IL-5 receptor $\alpha$ antibody, in a phase I study of subjects with mild asthma. J. Allergy Clin. Iimmunol., 125(6):1237-1244.e2. [doi:10.1016/j.jaci.2010.04.005]

Castro, M., Mathur, S., Hargreave, F., et al., 2011. Reslizumab for poorly controlled, eosinophilic asthma: a randomized, placebo-controlled study. Am. J. Respir. Crit. Care Med., 184(10):1125-1132. [doi:10.1164/rccm.201103-0396OC]

Cebrian, M., Yague, E., Rincon, M., et al., 1988. Triggering of $\mathrm{T}$ cell proliferation through AIM, an activation inducer molecule expressed on activated human lymphocytes. $J$. Exp. Med., 168(5):1621-1637. [doi:10.1084/jem.168.5. 1621]

Corren, J., Busse, W., Meltzer, E.O., et al., 2010. A randomized, controlled, phase 2 study of AMG 317, an IL-4R $\alpha$ antagonist, in patients with asthma. Am. J. Respir. Crit. Care Med., 181(8):788-796. [doi:10.1164/rccm.2009091448OC]

Cruz-Adalia, A., Jiménez-Borreguero, L., Ramírez-Huesca, M., et al., 2010. CD69 limits the severity of cardiomyopathy after autoimmune myocarditis. Circulation, 122(14): 1396-1404. [doi:10.1161/CIRCULATIONAHA.110.952820]

Dallaire, M.J., Ferland, C., Page, N., et al., 2003. Endothelial cells modulate eosinophil surface markers and mediator release. Eur. Respir. J., 21(6):918-924. [doi:10.1183/ 09031936.03.00102002] 
Epardaud, M., Elpek, K.G., Rubinstein, M.P., et al., 2008. Interleukin-15/interleukin-15R $\alpha$ complexes promote destruction of established tumors by reviving tumorresident $\mathrm{CD}^{+} \mathrm{T}$ cells. Cancer Res., 68(8):2972-2983. [doi:10.1158/0008-5472.CAN-08-0045]

Esplugues, E., Sancho, D., Vega-Ramos, J., et al., 2003. Enhanced antitumor immunity in mice deficient in CD69. J. Exp. Med., 197(9):1093-1106. [doi:10.1084/jem.2002 1337]

Gauvreau, G.M., Wood, L.J., Sehmi, R., et al., 2000. The effects of inhaled budesonide on circulating eosinophil progenitors and their expression of cytokines after allergen challenge in subjects with atopic asthma. Am. J. Respir. Crit. Care Med., 162(6):2139-2144. [doi:10.1164/ ajrccm.162.6.2001120]

Hamelmann, E., Schwarze, J., Takeda, K., et al., 1997. Noninvasive measurement of airway responsiveness in allergic mice using barometric plethysmography. Am. J. Respir. Crit. Care Med., 156(3):766-775. [doi:10.1164/ ajrccm.156.3.9606031]

Han, S.T., Mosher, D.F., 2014. IL-5 induces suspended eosinophils to undergo unique global reorganization associated with priming. Am. J. Respir. Cell Mol. Biol., 50(3):654-664. [doi:10.1165/rcmb.2013-0181OC]

Iwashiro, M., Messer, R.J., Peterson, K.E., et al., 2001. Immunosuppression by $\mathrm{CD}^{+}$regulatory $\mathrm{T}$ cells induced by chronic retroviral infection. PNAS, 98(16):9226-9230. [doi:10.1073/pnas.151174198]

Justice, J.P., Crosby, J., Borchers, M.T., et al., 2002. CD4 ${ }^{+} \mathrm{T}$ cell-dependent airway mucus production occurs in response to IL-5 expression in lung. Am. J. Physiol. Lung Cell. Mol. Physiol., 282(5):L1066-L1074. [doi:10.1152/ ajplung.00195.2001]

Lee, J.J., McGarry, M.P., Farmer, S.C., et al., 1997. Interleukin-5 expression in the lung epithelium of transgenic mice leads to pulmonary changes pathognomonic of asthma. J. Exp. Med., 185(12):2143-2156. [doi:10.1084/ jem.185.12.2143]

Letterio, J.J., Roberts, A.B., 1998. Regulation of immune responses by TGF- $\beta$. Annu. Rev. Immunol., 16(1):137-161. [doi:10.1146/annurev.immunol.16.1.137]

Lewkowich, I.P., Rempel, J.D., HayGlass, K.T., 2005. Prevention of allergen-specific, Th2-biased immune responses in vivo: role of increased IL-12 and IL-18 responsiveness. J. Immunol., 175(8):4956-4962. [doi:10. 4049/jimmunol.175.8.4956]

Luttmann, W., Matthiesen, T., Matthys, H., et al., 1999. Synergistic effects of interleukin-4 or interleukin-13 and tumor necrosis factor- $\alpha$ on eosinophil activation in vitro. Am. J. Respir. Cell Mol. Biol., 20(3):474-480. [doi:10. 1165/ajrcmb.20.3.3326]

Martin, P., Gomez, M., Lamana, A., et al., 2010a. CD69 association with Jak3/Stat5 proteins regulates Th17 cell differentiation. Mol. Cell. Biol., 30(20):4877-4889. [doi: 10.1128/MCB.00456-10]

Martin, P., Gomez, M., Lamana, A., et al., 2010b. The leukocyte activation antigen CD69 limits allergic asthma and skin contact hypersensitivity. J. Allergy Clin. Immunol., 126(2):355-365.e3. [doi:10.1016/j.jaci.2010. 05.010]

Matsumoto, K., Appiah-Pippim, J., Schleimer, R.P., et al., 1998. CD44 and CD69 represent different types of cell-surface activation markers for human eosinophils. Am. J. Respir. Cell Mol. Biol., 18(6):860-866. [doi:10. 1165/ajrcmb.18.6.3159]

McGee, H.S., Agrawal, D.K., 2006. $\mathrm{T}_{\mathrm{H}} 2$ cell in the pathogenesis of airway remodeling. Immunol. Res., 35(3):219-232. [doi:10.1385/IR:35:3:219]

Miki-Hosokawa, T., Hasegawa, A., Iwamura, C., et al., 2009. CD69 controls the pathogenesis of allergic airway inflammation. J. Immunol., 183(12):8203-8215. [doi:10. 4049/jimmunol.0900646]

Mohammadi Nejad, M., Salehi, E., Mesdaghi, M., et al., 2013. Increased expression of CD69 antigen on human peripheral blood natural killer cells in patients with allergic rhinitis. Iran. J. Allergy Asthma Immunol., 12(1): 68-74.

Pajusto, M., Ihalainen, N., Pelkonen, J., et al., 2004. Human in vivo-activated $\mathrm{CD} 45 \mathrm{R} 0^{+} \mathrm{CD}^{+} \mathrm{T}$ cells are susceptible to spontaneous apoptosis that can be inhibited by the chemokine CXCL12 and IL-2, -6, -7, and -15. Eur. J. Immunol., 34(10):2771-2780. [doi:10.1002/eji.200324761]

Pavord, I.D., Korn, S., Howarth, P., et al., 2012. Mepolizumab for severe eosinophilic asthma (DREAM): a multicentre, double-blind, placebo-controlled trial. Lancet, 380(9842): 651-659. [doi:10.1016/S0140-6736(12)60988-X]

Sancho, D., Gomez, M., Viedma, F., et al., 2003. CD69 downregulates autoimmune reactivity through active transforming growth factor- $\beta$ production in collageninduced arthritis. J. Clin. Invest., 112(6):872-882. [doi:10. 1172/JCI19112]

Santis, A.G., Campanero, M.R., Alonso, J.L., et al., 1992. Tumor necrosis factor- $\alpha$ production induced in $T$ lymphocytes through the AIM/CD69 activation pathway. Eur. J. Immunol., 22(5):1253-1259. [doi:10.1002/eji. 1830220521]

Sheikh Bahaie, N., Rao, S.P., Massoud, A., et al., 2010. GM-CSF differentially regulates eosinophil and neutrophil adhesive interactions with vascular endothelium in vivo. Iran. J. Allergy Asthma Immunol., 9(4):207-217.

Shen, H.H., Ochkur, S.I., McGarry, M.P., et al., 2003. A causative relationship exists between eosinophils and the development of allergic pulmonary pathologies in the mouse. J. Immunol., 170(6):3296-3305. [doi:10.4049/ jimmunol.170.6.3296]

Shinoda, K., Tokoyoda, K., Hanazawa, A., et al., 2012. Type II membrane protein CD69 regulates the formation of resting T-helper memory. PNAS, 109(19):7409-7414. [doi:10.1073/pnas.1118539109]

Spencer, L.A., Szela, C.T., Perez, S.A., et al., 2009. Human eosinophils constitutively express multiple Th1, Th2, and immunoregulatory cytokines that are secreted rapidly and 
differentially. J. Leukoc. Biol., 85(1):117-123. [doi:10. 1189/jlb.0108058]

Su, Y.C., Rolph, M.S., Hansbro, N.G., et al., 2008. Granulocyte-macrophage colony-stimulating factor is required for bronchial eosinophilia in a murine model of allergic airway inflammation. J. Immunol., 180(4):26002607. [doi:10.4049/jimmunol.180.4.2600]

Takatsu, K., Nakajima, H., 2008. IL-5 and eosinophilia. Curr. Opin. Immunol., 20(3):288-294. [doi:10.1016/j.coi.2008. 04.001]

Testi, R., D'Ambrosio, D., de Maria, R., et al., 1994. The CD69 receptor: a multipurpose cell-surface trigger for hematopoietic cells. Immunol. Today, 15(10):479-483. [doi:10. 1016/0167-5699(94)90193-7]

Tugores, A., Alonso, M.A., Sanchez-Madrid, F., et al., 1992. Human $\mathrm{T}$ cell activation through the activation-inducer molecule/CD69 enhances the activity of transcription factor AP-1. J. Immunol., 148(7):2300-2306.

Unutmaz, D., Pileri, P., Abrignani, S., 1994. Antigenindependent activation of naive and memory resting $\mathrm{T}$ cells by a cytokine combination. J. Exp. Med., 180(3): 1159-1164. [doi:10.1084/jem.180.3.1159]

van Rijt, L.S., Prins, J.B., Leenen, P.J., et al., 2002. Allergen-induced accumulation of airway dendritic cells is supported by an increase in $\mathrm{CD} 31^{\mathrm{hi}} \mathrm{Ly}-6 \mathrm{C}^{\text {neg }}$ bone marrow precursors in a mouse model of asthma. Blood, 100(10):3663-3671. [doi:10.1182/blood-2002-03-0673]

Vance, B.A., Harley, P.H., Backlund, P.S., et al., 2005. Human CD69 associates with an N-terminal fragment of calreticulin at the cell surface. Arch. Biochem. Biophys., 438(1):11-20. [doi:10.1016/j.abb.2005.04.009]

Vega-Ramos, J., Alari-Pahissa, E., Valle, J.D., et al., 2010. CD69 limits early inflammatory diseases associated with immune response to Listeria monocytogenes infection. Immunol. Cell Biol., 88(7):707-715. [doi:10.1038/icb. 2010.62]

Walsh, G.M., Williamson, M.L., Symon, F.A., et al., 1996. Ligation of CD69 induces apoptosis and cell death in human eosinophils cultured with granulocyte-macrophage colony-stimulating factor. Blood, 87(7):2815-2821.
Wang, H.Y., Shen, H.H., Lee, J.J., et al., 2006. CD69 expression on airway eosinophils and airway inflammation in a murine model of asthma. Chin. Med. J. (Engl.), 119(23): 1983-1990.

Zhao, Q., Kuang, D.M., Wu, Y., et al., 2012. Activated CD69 ${ }^{+}$ $\mathrm{T}$ cells foster immune privilege by regulating IDO expression in tumor-associated macrophages. J. Immunol., 188(3):1117-1124. [doi:10.4049/jimmunol.1100164]

\section{中文概要}

题 目: 抗 CD69 单克隆抗体对哮喘小鼠的气道炎症抑制 作用研究

目 的: 气道慢性炎症和高反应性是哮喘最主要的病理生 理表现, CD69 是一种与免疫细胞活化、增殖及 调亡密切相关的共刺激分子, 本研究旨在观察抗 CD69 单克隆抗体在哮喘小鼠模型中抑制气道炎 症和高反应性的作用及机制探讨。

创新点: (1) 与大多数关于哮喘的药物研究多为造模前提 前用药以阻断炎症的进展相比, 本研究将抗 CD69 单抗作用于气道己经形成的炎症, 与临床 上哮喘的治疗更接近; (2) 抗 CD69 单抗的独特 作用在于它特异性地作用于气道活化的炎症细 胞, 与已有的哮喘药物以糖皮质激素相比, 可能 的全身反应少。

方 法: 鸡卵白蛋白致敏激发制备哮喘模型 (图 1), Buxco 系统测试哮喘小鼠的气道高反应性（图 6)。

结 论: 抗 CD69 单克隆抗体可抑制哮喘小鼠已经形成的 气道炎症，其作用效果与地塞米松预处理相当。 本研究为临床哮喘的急性发作治疗和管理提供 了新的可能靶点。

关键词：分化抗原簇 69 (CD69); 白介素-5; 哮喘; 嗜酸 性粒细胞 